\title{
Effects of hypoxia on differentiation of menstrual blood stromal stem cells towards tenogenic cells in a co-culture system with Achilles tendon cells
}

\author{
YIJING ZHENG $^{1 *}$, YIFEI ZHOU ${ }^{1 *}$, XIAOLEI ZHANG ${ }^{1}$, YUEMIAO CHEN $^{2}$,

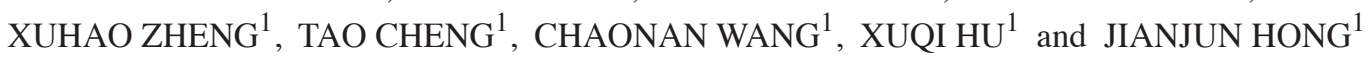

${ }^{1}$ Department of Orthopedics, The Second Affiliated Hospital and Yuying Children's Hospital of Wenzhou Medical University,
Wenzhou, Zhejiang 325027; ${ }^{2}$ Department of Hematology, Wenzhou Central Hospital, Wenzhou, Zhejiang 325000, P.R. China

Received, August 11, 2015; Accepted December 9, 2016

DOI: $10.3892 / \mathrm{etm} .2017 .4383$

\begin{abstract}
Achilles tendons have a very poor capacity for intrinsic regeneration. The cell-based treatment strategy for Achilles tendinitis includes the application of mesenchymal stem cells (MSCs), which have high proliferative and multipotent differentiation ability, and is a promising approach. The aim of the present study was to explore the tenogenic potential of human menstrual blood stromal stem cells (MenSCs) in a co-culture system and to compare the tenogenic capability under normoxic and hypoxic conditions. MenSCs were co-cultured indirectly with Achilles tendon cells in a Transwell co-culture system for 1,2 , or 3 weeks in two different concentrations of oxygen (20 and 2\% $\mathrm{O}_{2}$ ), whereas the control contained only MenSCs. The extracellular matrix of MenSCs in each system was evaluated by Alcian blue staining assay, histological staining, reverse transcription-quantitative polymerase chain reaction (RT-qPCR), and western blot analysis. Alcian blue staining assay revealed a significant increase $(\mathrm{P}<0.05)$ in proteoglycan secretion by the differentiated MenSCs. Identical results were obtained by RT-qPCR for collagen I, which was validated by western blot analysis. Considerably increased collagen I and collagen III gene expression levels were exhibited by cells in the co-culture treatment group when compared with the control $(\mathrm{P}<0.05)$; however, no significant difference was detected between the normoxic $\left(20 \% \mathrm{O}_{2}\right)$ and hypoxic treatment $\left(2 \% \mathrm{O}_{2}\right)$ groups.
\end{abstract}

Correspondence to: Professor Jianjun Hong, Department of Orthopedics, The Second Affiliated Hospital and Yuying Children's Hospital of Wenzhou Medical University, 109 Western Xueyuan Road, Wenzhou, Zhejiang 325027, P.R. China

E-mail: hjjwygk@126.com

*Contributed equally

Key words: Achilles tendinitis, Achilles tendon cell, co-culture, differentiation, hypoxia, menstrual blood stromal stem cells
RT-qPCR was utilized to determine the expression levels of thrombospondin 4, scleraxis and tenascin $\mathrm{C}$ in the differentiated MenSCs; a significant increase in the expression of these specific genes was indicated in the co-culture treatment group compared with the control $(\mathrm{P}<0.05)$. Although the expression levels were markedly higher in hypoxia than in normoxia conditions, this difference was not significant. To conclude, the present study indicated that MenSCs manifested a strong proliferative and multipotent capacity for differentiation and differentiated into Achilles tenogenic cells. Therefore, the use of MenSCs may be considered in Achilles tendinitis therapy.

\section{Introduction}

A number of people, particularly those who have engaged in sports activities, often experience heel pain. Achilles tendinitis is generally recognized to be the predominant cause of heel pain (1). Typically, the treatment methods applied at present, such as surgical or non-operative therapy, exert limited effects in treating the symptoms, rather than targeting the root cause of Achilles tendinitis.

Achilles tendinitis is considered a non-inflammatory condition and has been characterized as either lipoid or mucoid degeneration (2). During this process, the normal white, glistening appearance of the tendon is lost, and it becomes grayish or brown and is mechanically softer (2). In addition to its color and substance changes, the damaged Achilles tendon is characterized by increased vascularization or neovascularization (3). These alterations lead to an abrupt reduction in the formation of matrix components, such as collagen type I (Col I), collagen type III (Col III), subsequently, substantial impairment is inflicted on the tendon, and eventually Achilles tendinitis occurs (4).

Previous reports have suggested that the application of stem cells can accelerate the healing of the Achilles tendon in animal models $(5,6)$. Due to the proliferation capacity and pluripotentiality of MSCs, the effects of the transplantation of stem cells may be exerted not only through direct differentiation of the mesenchymal stem cells, but also via the release of paracrine factors, such as growth factors and cytokines, secreted by the cells (7). In previous analysis, the most common types of 
MSCs utilized in experiments are bone marrow mesenchymal stem cells (BMSCs) (8) and adipose-deprived mesenchymal stem cells (9). However, certain disadvantages in the application of these adult stem cells have been identified, including the invasiveness of the procedures for the collection of MSCs and the poor potential for proliferation, which is lower than that of embryonic stem cells (10).

Stromal stem cells obtained from menstrual blood (MenSCs) have been utilized as a novel source in previous studies $(11,12)$, demonstrating important advantages, such as a decreased hazard of anomalies in the karyotype and a minimized possibility for tumor formation and acceleration in animal models (13). MenSCs are derived by non-invasive methods, thus this cell type is ethically suitable and autologous. Therefore, MenSCs can be considered the perfect stem cell type for the treatment of Achilles tendinitis.

In previous research, tendon genes, such as Col I and Col III, were typically used to assess the tenogenic differentiation of MSC. Specific human tendon genes, including thrombospondin 4 (THBS4), scleraxis (Scx), and tenascin C (TENC), were identified through a comparison between the gene expression rates of tenogenic cells and Achilles tendon cells (14). However, little is known on the exact MSC commitment process and the mechanism of differentiation. The present study aimed to establish a co-culture system of MenSCs and Achilles tendon cells in normoxia $\left(20 \% \mathrm{O}_{2}\right)$ and hypoxia $(2 \%$ $\mathrm{O}_{2}$ ) conditions, respectively. The extracellular matrix (ECM) and the distinctive human tendon genes were utilized to assess the tenogenic differentiation of MenSCs.

\section{Materials and methods}

Ethics statement. The present study was conducted according to the tenets of the Declaration of Helsinki. All patients were candidates for Achilles tendon surgical repair and gave their written informed consent and approved the use of the resected Achilles tendon from both broken ends for further basic research. The study design and the protocol had been granted a written agreement by the Second Affiliated Hospital of Wengzhou Medical University Research Ethics Committee. In the experiments the part of the Achilles tendon that required removal before suture was used, which prevented any secondary damage to the tissue.

Culture of Achilles tendon cells (ATCs). Between January and June 2012, tissue samples from the Achilles tendons were collected from 3 patients (2 male, 1 female; age, 23-45 years), suffering from closed Achihes tendon rupture, during surgery. Patients exhibited no underlying health problems or serious medical conditions. Tissues were washed with phosphate-buffered saline (PBS) and digested enzymatically with $0.2 \%$ collagenase type II (Sigma-Aldrich; Merck Millipore, Darmstadt, Germany) at $37^{\circ} \mathrm{C}$ for $6 \mathrm{~h}$. Subsequently, the tissue debris was removed by passage through a $200-\mu \mathrm{m}$ filter and the obtained ATCs were centrifuged at $250 \mathrm{x} g$ for $10 \mathrm{~min}$ at $4^{\circ} \mathrm{C}$ and resuspended in Dulbecco's modified Eagle's medium (DMEM) and Ham's F-12 Nutrient Mixture, supplemented with $10 \%$ fetal bovine serum (FBS) (all Gibco; Thermo Fisher Scientific Inc., Waltham, MA, USA) and $1 \%$ penicillin/streptomycin (Beijing Solarbio Science \& Technology, Beijing,
China). The suspension was incubated at $37^{\circ} \mathrm{C}$ in a humidified atmosphere containing $5 \% \mathrm{CO}_{2}$. The medium was changed 2-3 times a week. Cells were observed under a light microscope (magnification, x100).

Culture of human MenSCs. Human MenSCs were purchased from S-Evans Biosciences (Hangzhou, China). Identification of cell surface markers, such as cell differentiation (CD)-73, CD-90, CD-105, CD-14, CD-19, CD-34, CD-35, and human leucocyte antigen antigen D-related (HLA-DR) were examined by $\mathrm{S}$-Evans Biosciences with flow cytometry. The cells were cultured in DMEM/F-12 media and 10\% FBS (both Gibco; Thermo Fisher Scientific, Inc.); then, they were incubated at a $37^{\circ} \mathrm{C}$, in a $5 \% \mathrm{CO}_{2}$ environment.

Co-culture of MenSCs and ATCs. A co-culture system was constructed with a Transwell chamber (Corning Inc., Corning, NY, USA), which could be inserted into the wells of 6-well plates. MenSCs were seeded on 6-well tissue culture polystyrene plates (Corning Inc.) at a density of $1 \times 10^{4}$ cells/well, whereas ATCs were seeded at the ratio of 1:1 with MenSCs on the membrane (polyethylene terephthalate, pore size, $0.4 \mathrm{~mm}$ ) of the Transwell chamber. ATCs and MenSCs were incubated for 1,2 , and 3 weeks at $37^{\circ} \mathrm{C}$ in a humidified atmosphere with either $95 \%$ air $\left(21 \% \mathrm{O}_{2}\right)$ plus $5 \% \mathrm{CO}_{2}$ (designated as normoxia) or with $2 \% \mathrm{O}_{2}$ plus $5 \% \mathrm{CO}_{2}$ and $93 \% \mathrm{~N}_{2}$ (low $\mathrm{O}_{2}$ tension) with a medium change every $2-3$ days. MenSCs $\left(1 \times 10^{4}\right)$ cultured in 6-well plates without ATCs were used as the control group. The culture medium was replaced every 3 days.

Alcian blue staining. Following co-culture for 2 weeks, MenSCs from each group were fixed in 4\% paraformaldehyde (PFA), washed with PBS and stained with $0.5 \%$ Alcian blue (Sigma-Aldrich; Merck Millipore) in 3\% acetic acid ( $\mathrm{pH} 2.5$ ) for $30 \mathrm{~min}$. Cells were visualized and images were captured using light microscopy (magnification, x200; Leica DM 2500; Leica Microsystems GmbH, Wetzlar, Germany). Image-Pro Plus (IPP) software (version 6.0; Media Cybernetics, Inc., Rockville, MD, USA) was used to evaluate the mean stain density of each image.

Immunohistochemical staining. Glass coverslips $(0.17 \mathrm{~mm})$ with MenSCs following 1, 2, and 3 weeks of co-culture were fixed with 4\% PFA in PBS at room temperature for $20 \mathrm{~min}$ and washed with PBS. Following permeation with Triton X-100 for $10 \mathrm{~min}$ and inhibition of endogenous peroxidase activity with $3 \%$ hydrogen peroxide for $15 \mathrm{~min}$. MenSCs were blocked with $5 \%$ goat albumin in PBS for $30 \mathrm{~min}$ at $37^{\circ} \mathrm{C}$. Subsequently, cells were incubated with an antibody against THBS4 (rabbit polyclonal; 1:800 dilution; sc-7657-R; Santa Cruz Biotechnology, Inc., Dallas, TX, USA) at $4^{\circ} \mathrm{C}$ overnight and washed with PBS 3 times, followed by incubation with a horseradish peroxidase-conjugated secondary antibody (1:200 diltuion; PV-6001; ZSGB-BIO, Beijing, China) at $37^{\circ} \mathrm{C}$ for 30 min. A DAB detection kit (Dako North America, Inc., Carpinteria, CA, USA) was utilized until desired staining intensity developed, and the cells were counterstained with hematoxylin. Subsequently, the samples were observed under a light microscope at a magnification of x10 (Leica DM 2500). Quantitative analysis of staining was performed using IPP 
Table I. Primer sequences for reverse transcription-quantitative polymerase chain reaction.

\begin{tabular}{lll}
\hline Target gene & Direction & Primer sequences \\
\hline Collagen I & Forward & 5'GAGGGCCAAGACGAAGACATC 3 \\
Collagen III & Reverse & 5'CAGATCACGTCATCGCACAAC 3' \\
Thrombospondin 4 & Forward & 5'GGAGCTGGCTACTTCTCGC 3' \\
& Reverse & 5'GGGAACATCCTCCTTCAACAG 3' \\
Tenascin C & Forward & 5'TGCTGCCAGTCCTGACAGA 3' \\
& Reverse & 5'GTTTAAGCGTCCCATCACAGTA 3' \\
Scleraxis & Forward & 5'TCCCAGTGTTCGGTGGATCT 3' \\
& Reverse & 5'TTGATGCGATGTGTGAAGACA 3' \\
18S rRNA & Forward & 5'AGAACACCCAGCCCAAACAG 3' \\
& Reverse & 5'TTGCTCAACTTTCTCTGGTTGCT 3' \\
& Forward & 5'GATGCGGCGGCGTTATTC 3' \\
\hline
\end{tabular}

software (version 6.0; Media Cybernetics, Inc.) to evaluate the mean stain density of each image.

Quantitative real-time polymerase chain reaction $(R T-q P C R)$. RT-qPCR was used to quantify the mRNA expression of Col I, Col III, THBS4, TENC, and Scx. RNA was isolated from each group by using TRIzol reagent (Invitrogen; Thermo Fisher Scientific Inc.) according to the manufacturer's instructions and the concentration of total RNA was determined using a spectrophotometer (DU-500; Beckman Coulter, Inc., Fullerton, CA, USA) at $260 \mathrm{~nm}$ wavelength. First-strand cDNA was synthesized in a $20 \mu \mathrm{l}$ reaction system containing: $11 \mu \mathrm{l} \mathrm{DEPC} \mathrm{ddH}_{2} \mathrm{O}$ with total RNA $(2 \mu \mathrm{g}), 1 \mu \mathrm{l}$ Oligo (dT) 18 primer, $4 \mu \mathrm{l}$ 5x Reaction buffer, $2 \mu 110 \mathrm{mM}$ dNTP mix, $1 \mu$ l Ribolock RNase inhibitor, and $1 \mu \mathrm{l}$ RevertAid M-MULV reverse transcriptase (MBI Fermentas, Sankt Leon-Rot, Germany). qPCR was performed in triplicate using a CFX96 Real-Time PCR system (Bio-Rad Laboratories, Inc., Hercules, CA, USA) under the following conditions: $10 \mathrm{~min}$ at $95^{\circ} \mathrm{C}$, followed by 40 cycles of $15 \mathrm{sec}$ at $95^{\circ} \mathrm{C}$ and $1 \mathrm{~min}$ at $60^{\circ} \mathrm{C}$ for $\mathrm{qPCR}$ amplification. The reaction was performed in a total volume of $20 \mu \mathrm{l}$, containing $10 \mu \mathrm{l}$ SYBR Green Master Mix (Bio-Rad Laboratories, Inc.), $2 \mu 1$ cDNA, $1 \mu \mathrm{l}$ each primer and $6 \mu \mathrm{l}$ sterile distilled water. Target gene expression was calculated using the $2^{-\Delta \Delta \mathrm{Cq}}$ method (15) and normalized to $18 \mathrm{~S}$ ribosomal RNA. The primers used are indicated in Table I.

Western blot analysis. Cells from different groups were harvested and lysed in a standard buffer (Beyotime Institute of Biotechnology, Haimen, China), and the total protein was extracted by using a Western and IP Cell Lysis kit (Beyotime Institute of Biotechnology). Cell lysates were separated and collected by centrifugation at $12,000 \mathrm{x} \mathrm{g}$ for $10 \mathrm{~min}$ at $4^{\circ} \mathrm{C}$. Protein concentrations were determined using an enhanced bicinchoninic acid assay kit (Beyotime Institute of Biotechnology). Equal quantities (30 $\mu \mathrm{g})$ of protein from each sample were separated by $10 \%$ SDS PAGE and transferred onto nitrocellulose membranes. Following washing three times with TBS containing $0.1 \%$ Tween-20 for $5 \mathrm{~min}$, non-fat milk was utilized to block non-specific blinding and the membranes were incubated overnight at $4^{\circ} \mathrm{C}$ with a rabbit polyclonal antibody conjugated with THBS4 (rabbit polyclonal; 1:1,000 dilution; sc-7657-R; Santa Cruz Biotechnology, Inc.), COL I (goat polyclonal; 1:600 dilution; sc-8784; Santa Cruz Biotechnology, Inc.), and GAPDH (rabbit polyclonal; 1:1,000 dilution; BS60630; Bioworld Technology, Inc., St. Louis Park, MN, USA). Following washing three times with TBS containing $0.1 \%$ Tween-20 for $5 \mathrm{~min}$ and incubation for $2 \mathrm{~h}$ at room temperature with the following horseradish peroxidase-conjugated secondary antibodies: Rhodamine (TRITC), AffiniPure Rabbit Anti-Goat IgG (CW0168) and Goat anti Rabbit IgG (CW0111; all 1:3,000 dilution; Beijing ComWin Biotech Co., Ltd., Beijing, China); the membranes were treated with enhanced chemiluminescence (ECL) Plus reagent (Bio-Rad Laboratories, Inc.), followed by detection using the ChemiDoc XRS+ system with Quantity ONE software $(1,708,265$; Bio-Rad Laboratories, Inc.) software.

Statistical analysis. Data are expressed as the mean \pm standard deviation. Statistical evaluation of the differences between and within the groups was performed using one-way analysis of variance followed by post-hoc Tukey's test. $\mathrm{P}<0.05$ was considered to indicate a statistically significant difference.

\section{Results}

Cell surface markers. As shown in Fig. 1, the following multipotent surface markers are present on mesenchymal stem cells: CD73, CD90, and CD105; whereas CD14, CD19, CD34, CD35, and HLA-DR were absent on these cells, which were also absent in the cells identified by Cui et al (16). Furthermore the MenSCs appear to have similar characteristics as the human endometrial stem cells identified by Cho et al (17). The expression of multipotent markers is indicative of cells that have the capacity to differentiate into cell types derived from multiple germ layers.

Alcian blue staining. Following 2 weeks of co-culture, Alcian blue staining was applied to stain the proteoglycan secreted by MenSCs. MenSCs from the control treatment were stained negatively (Fig. 2A and B). Conversly, staining of MenSCs 


$\begin{array}{ccc}\text { A Surface marker } & \text { Normal standard } & \text { Result } \\ \text { CD73 } & \geq 95 \% & 99.7 \% \\ \text { CD90 } & \geq 95 \% & 98.0 \% \\ \text { CD105 } & \geq 95 \% & 99.3 \% \\ \text { CD34 } & \leq 2 \% & 0.3 \% \\ \text { CD45 } & \leq 2 \% & 1.2 \% \\ \text { CD14 } & \leq 2 \% & 0.5 \% \\ \text { CD19 } & \leq 2 \% & 0.8 \% \\ \text { HLA-DR } & \leq 2 \% & 0.7 \%\end{array}$

\section{B}

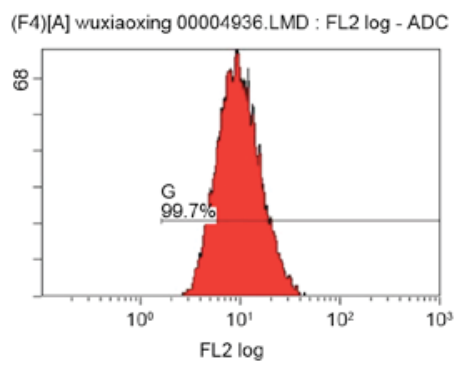

\section{$\mathbf{E}$}

(F1)[A] wuxiaoxing 00007042.LMD : FL2 log - ADC

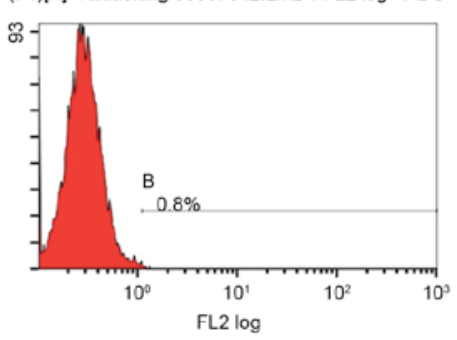

C

(F2)[A] wuxiaoxing 00007026.LMD : FL2 log - ADC

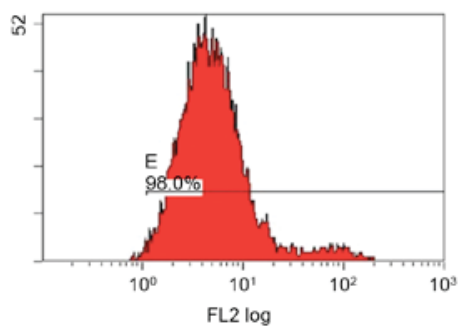

$\mathbf{F}$

(F1)[A] wuxiaoxing 00007042.LMD : FL2 log - ADC

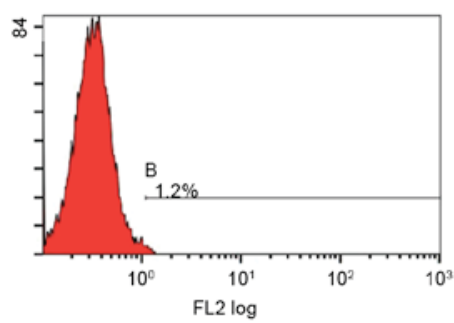

\section{D}

(F2)[A] wuxiaoxing 00004934.LMD : FL2 log - ADC

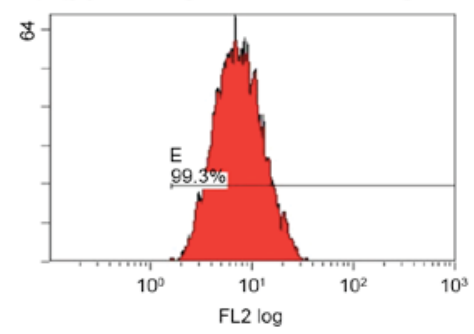

G

(F1)[A] wuxiaoxing 00007042.LMD : FL2 log - ADC

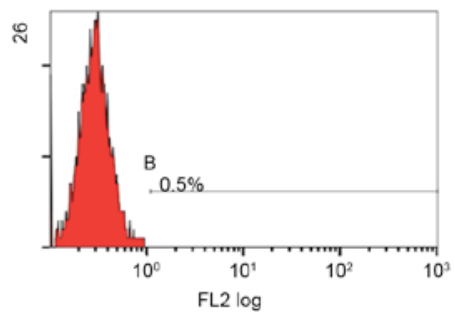

H

(F1)[A] wuxiaoxing 00007042.LMD : FL2 log - ADC

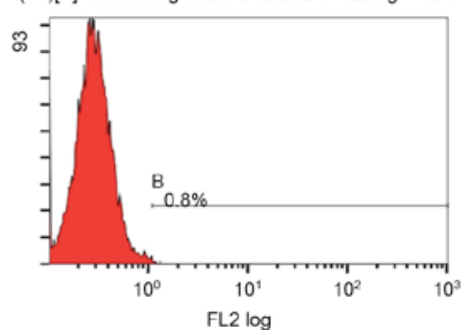

I

(F1)[A] wuxiaoxing 00007042.LMD : FL2 log - ADC

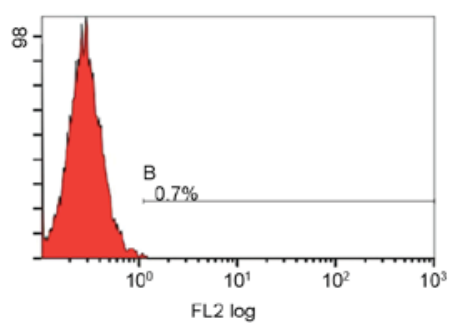

Figure 1. (A-I) Flow cytometric analysis of MenSCs. The (A) results of flow cytometry showed that MenSCs were positive for (B) CD73, (C) CD90 and (D) CD105, and negative for (E) CD14, (F) CD19, (G) CD34, (H) CD35, and (I) HLA-DR. MenScs, menstrual blood stromal cells; CD, cluster of differentiation; HLA-DR, human leucocyte antigen antigen D-related.

was substantially promoted, and Alcian blue metachromasia was evident in MenSCs under hypoxic (Fig. 2C) and normoxic (Fig. 2D) conditions. According to Fig. 2E, higher proteoglycan secretion was exhibited in the hypoxic cell group than in the normoxic group; however, no significant difference was established between these groups $(\mathrm{P}>0.05)$.

Immunohistochemical staining. THBS4 immunohistochemical staining was implemented to identify the level of THBS4 secretion by MenSCs. Following co-culture with ATCs, MenSCs from the control group were stained negatively, whereas MenSCs in the hypoxic treatment group were stained more intensely than those in the normoxic group (Fig. 3A-H).
The results were more obvious in Fig. 3I, which was processed by IPP 6.0 .

$R T$ - $q P C R$. Following co-culture, RT-qPCR was performed to analyze the expression of ECM genes (collagen I, collagen III, THBS4, scleraxis, and tenascin C). By the end of the 3-week co-culture, gene expression levels of Col I, Col III, THBS4, TENC, and Scx in the normoxic and hypoxic groups were significantly higher than those in the control group $(\mathrm{P}<0.05$; Fig. 4A-E, respectively). Moreover, the expression of ECM genes within the normoxic and hypoxic treatments were significantly upregulated $(\mathrm{P}<0.05$; Fig. 4). Nevertheless, the expression of Col I, Col III, THBS4, TENC, and Scx in the 

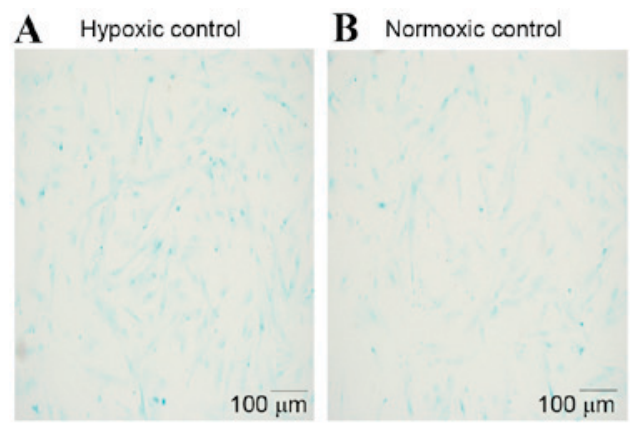

C Hypoxic group

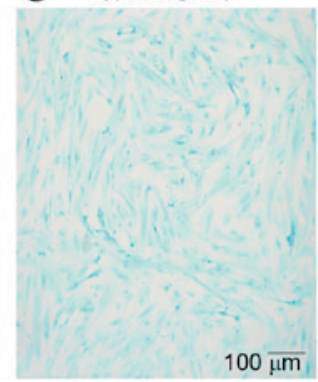

D Normoxic group

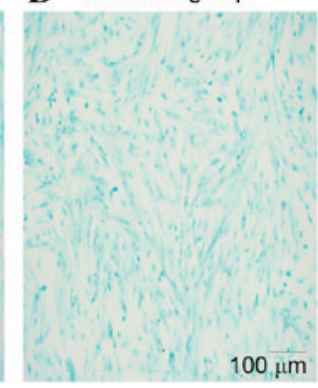

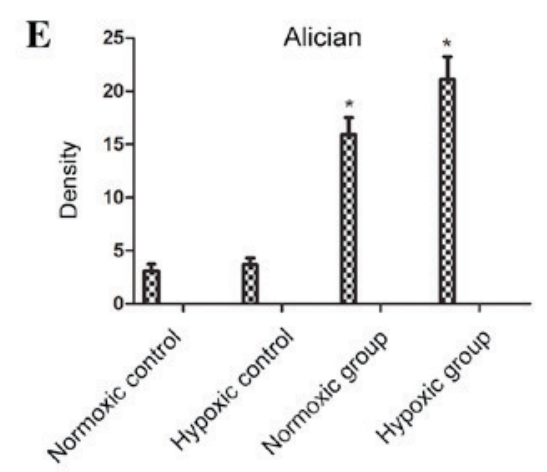

Figure 2. Alcian blue staining of MenSCs. MenSCs were co-cultured indirectly with Achilles tendon cells in a Transwell co-culture system in two different concentrations of oxygen $\left(20 \% \mathrm{O}_{2}\right.$, normoxic conditions and $2 \% \mathrm{O}_{2}$, hypoxic conditions), whereas the control contained only MenSCs. Control MenSCs under (A) hypoxic and (B) normoxic conditions. MenSCs under (C) hypoxic and (D) normoxic conditions. The co-culture group exhibited a higher intensity metachromatic effect when compared with the control group. (E) Alcian staining density of each group. Data are presented as the mean + standard deviation. ${ }^{*} \mathrm{P}<0.05$ vs. control. Scale bar, $100 \mu \mathrm{m}$ (magnification, $\mathrm{x} 10$ ). MenSCs, menstrual blood stromal stem cells.
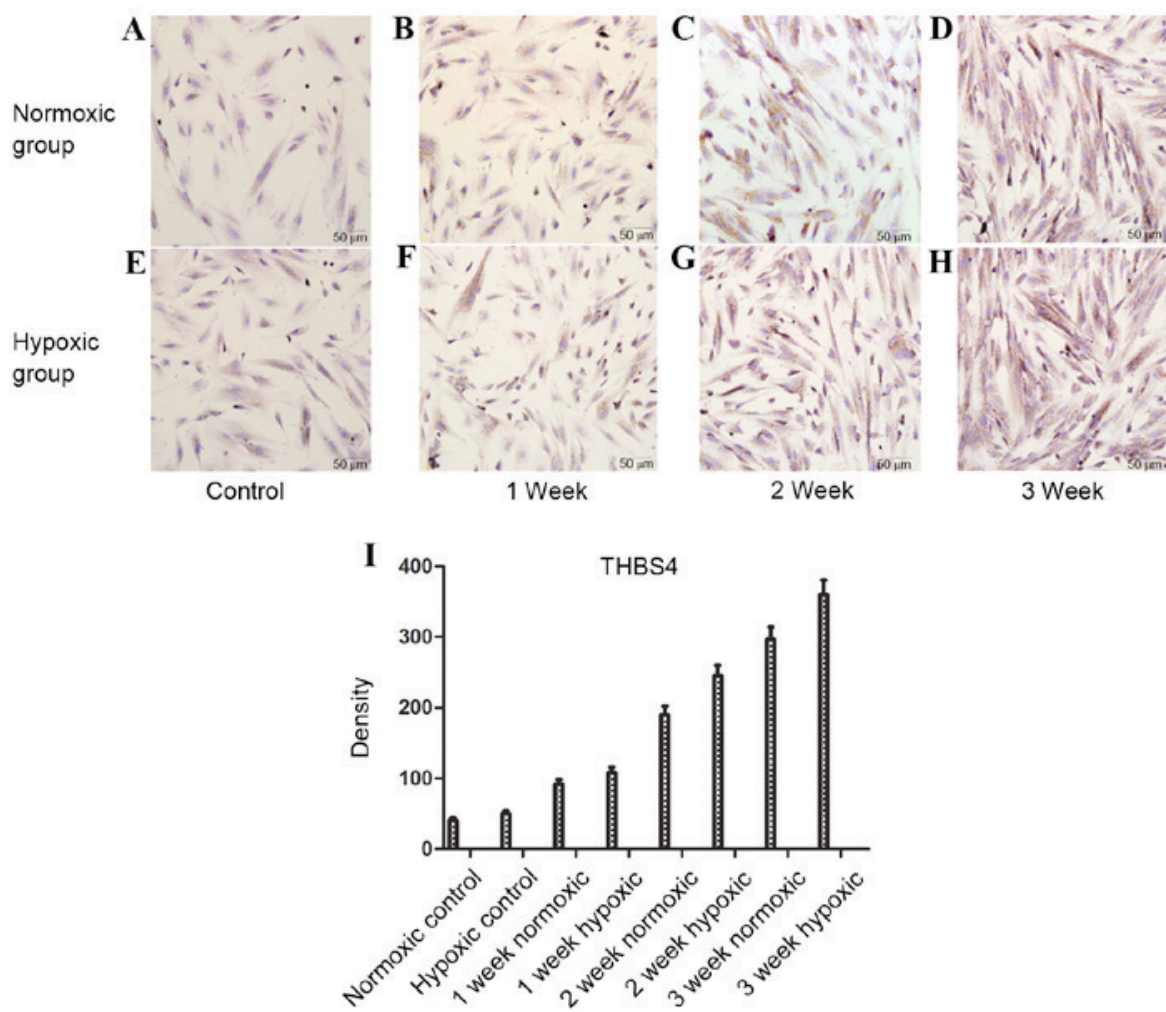

Figure 3. Immunohistochemical staining of MenSCs. Immunohistochemical staining revealed the secretion of THBS4 by MenSCs. MenSCs were co-cultured indirectly with Achilles tendon cells in a transwell co-culture system, for 1,2 , or 3 weeks in two different concentrations of oxygen $\left(20 \% \mathrm{O}_{2}\right.$ and $\left.2 \% \mathrm{O}_{2}\right)$, whereas the control contained only MenSCs. (A) Normoxic control. (B) Normoxic MenSCs co-cultured for 1 week. (C) Normoxic MenSCs co-cultured for 2 weeks. (D) Normoxic MenSCs co-cultured for 3 weeks. (E) Hypoxic control. (F) Hypoxic MenSCs co-cultured for 1 week. (G) Hypoxic MenSCs co-cultured for 2 weeks. (H) Hypoxic MenSCs co-cultured for 3 weeks. (Scale bar $100 \mu \mathrm{m}$ ). (I) THBS4 staining density of each group, quantified by Image Pro Plus 6.0 software. THBS4 immunohistochemical staining was positive, showing a strong color in normoxic and hypoxic groups (B, C, D, F, G, and H: color intensity $\mathrm{D}>\mathrm{C}>\mathrm{B} ; \mathrm{H}>\mathrm{G}>\mathrm{F} ; \mathrm{F}>\mathrm{B} ; \mathrm{G}>\mathrm{C} ; \mathrm{H}>\mathrm{D}$ ), whereas it was not obvious in the control group (A and E). Data are presented as the mean + standard deviation. MenSCs, menstrual blood stromal stem cells; THBS4, thrombospondin 4. 
A

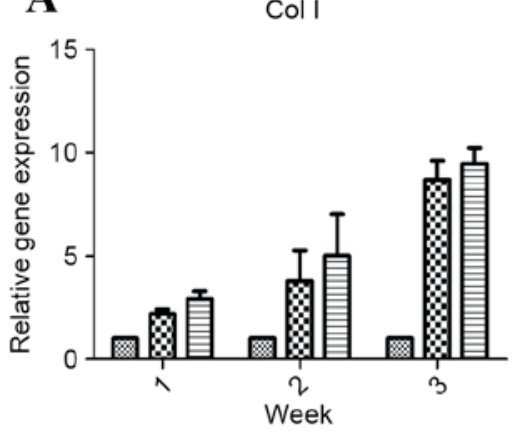

C

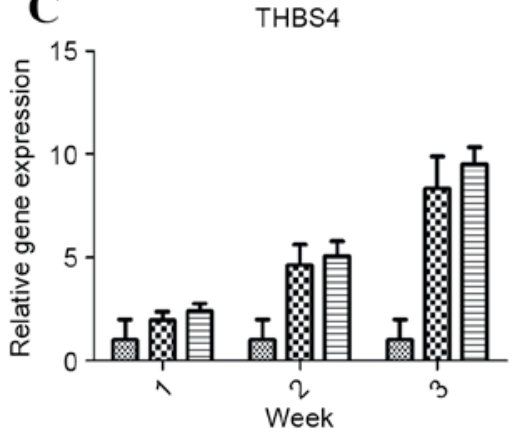

B

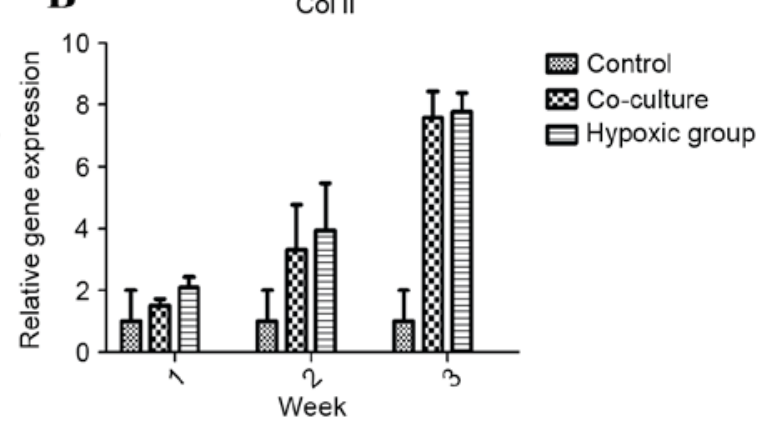

D

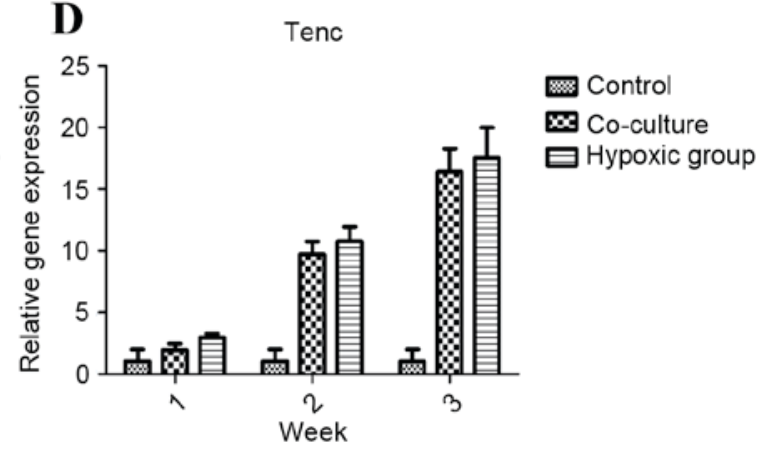

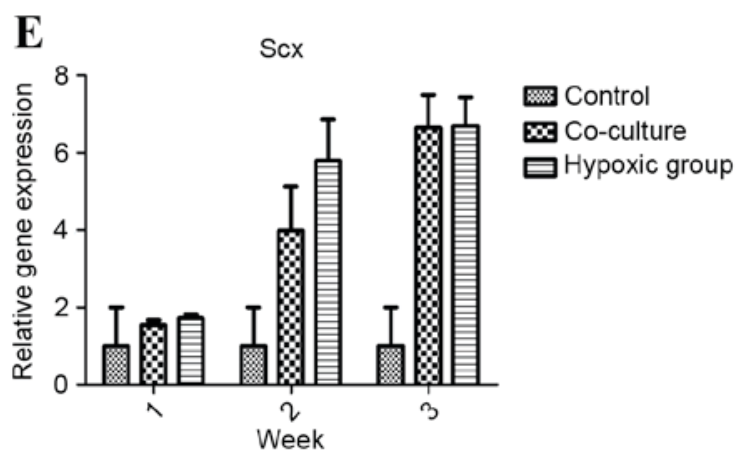

Figure 4. Extracellular matrix genes expression of MenSCs determined by reverse transcription-quantitative polymerase chain reaction. Expression levels of (A) Col I, (B) Col III, (C) THBS4, (D) TENC and (E) Scx mRNA in MenSCs following co-culture. Expression levels of each gene were normalized to 18S rRNA and control; $n=3$. Data are presented as the mean + standard deviation. MenSCs, menstrual blood stromal stem cells; Col I, collagen type I; Col III, collagen type III; THBS4, thrombospondin 4; Scx, scleraxis; TENC, tenascin C.

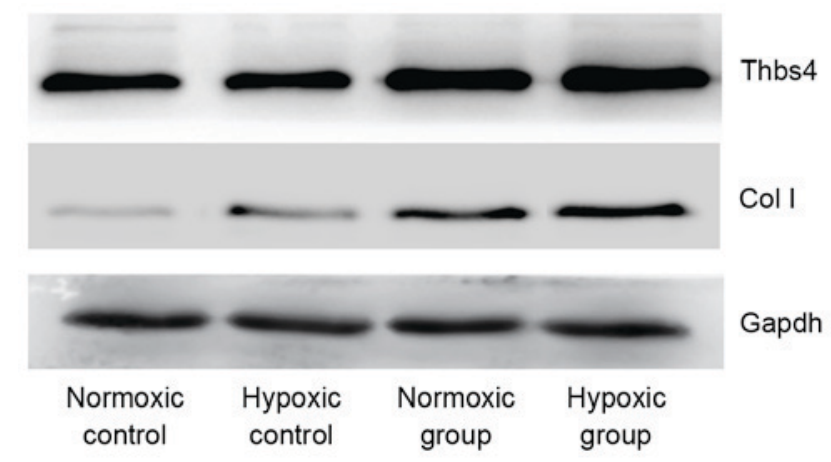

Figure 5. Western blot analysis of MenSCs. Western blotting of Col I and THBS4 protein in MenSCs was performed following 3-week co-culture. The amounts of these proteins in the hypoxic and normoxic treatments were greater than those in the control group. However, there was no marked difference between the quantities of the proteins mentioned in the cells under hypoxia and those under normoxia. GAPDH was used as a charge control. MenSCs, menstrual blood stromal stem cells; Col I, collagen type I; THBS4, thrombospondin 4; GAPDH, glyceraldehyde 3-phosphate dehydrogenase. normoxic cell group did not differ significantly when compared with the hypoxic group ( $\mathrm{P}>0.05$; Fig. 4A-E, respectively).

Western blot analysis. Western blotting was applied to detect $\mathrm{Col} I$ and THBS4 protein and quantify their content. Following 3 weeks of co-culture, Col I and THBS4 proteins were present in all groups. Quantities of these proteins in the hypoxic and normoxic groups were higher when compared with the control group. However, there was no obvious difference between the quantities of these proteins in the hypoxia group and the normoxia group (Fig. 5).

\section{Discussion}

Owing to the extremely poor self-healing capacity of the human tendon, Achilles tendinitis has long been a clinical challenge $(18,19)$. Transplantation of MSCs provides an excellent solution for the treatment of Achilles tendinitis. Through 
the implementation of this method, tenogenic differentiation is achieved and supplementation of the ECM is promoted, which stimulates the recovery of the degenerated tendon and leads to its healing.

Previous findings indicate that MenSCs, a new source of MSCs, have been successfully differentiated into chondrogenic, adipogenic, osteogenic (20), neurogenic, and cardiogenic cell lineages (12). The results of these studies demonstrate the plasticity of MenSCs for potential research in regenerative medicine. However, it is remains uncertain whether this cell type is able to differentiate into tenogenic cell lineages. The present study examined for the first time the in vitro capacity for tenogenic differentiation of MenSCs. ATCs are located in a comparatively avascular tissue area, and their development is hindered by the phenotype drift and loss of functions (21). An exceedingly low number of arterioles in proximity to the Achilles tendon have been established in an examination of the local blood supply, which indicated that the low oxygen environment is the natural medium of the Achilles tendon (22). We hypothesized that the level of oxygen exerted an effect on the differentiation of MenSCs into tenogenic cells as ATCs exist and operate under hypoxic conditions. There is evidence that oxygen tension of $2 \%$ enhanced the differentiation of human MSCs into cells similar to nucleus pulposus cells (23) and chondrocytes (24). Thus, low oxygen tension $\left(2 \% \mathrm{O}_{2}\right)$ was utilized to determine whether hypoxia was able to stimulate the differentiation of MenSCs into tenogenic cells. In the present study, the results of Alcian blue Staining revealed that the secretion of proteoglycan increased significantly in the differentiated MenSCs obtained from both co-culture groups. RT-qPCR analysis indicated identical findings for Col I, which was also confirmed by western blotting. Gene expression of Col I and Col III in the co-culture group were significantly higher when compared with the control group, which is important for maintaining the function of the Achilles tendon. However, no significant difference was observed between the normoxic and hypoxic groups. Furthermore, RT-qPCR revealed significant upregulation of the expression of the specific tenogenic genes (THBS4, scleraxis, and tenascin C) in the differentiated MenSCs. However, when compared with the normoxia group, the expression levels were slightly higher in the hypoxia group. These findings are not consistent with the data from previous studies, which indicated that hypoxia substantially increased ECM synthesis of differentiated MSCs (25). This discrepancy may be a result of the living environment of MenSCs, as they are located in the endometrium, which is characterized by an abundant blood supply, whereas the oxygen concentration of bone marrow is $\sim 1-7 \%$ (26). Thus, MenSCs had to adapt to the $2 \% \mathrm{O}_{2}$ environment when they were cultured. In future research, pre-incubation of MenSCs in a $2 \% \mathrm{O}_{2}$ environment or prolonged co-culture time may further promote tenogenic differentiation of MenSCs in hypoxic conditions.

The large pentameric glycoprotein, THBS4, which is a tendon-selective marker, can bind to $\mathrm{Col}$ II in the tendon tissue and form complex compounds with the cartilage oligomeric matrix protein (27). The expression of THBS4 is highly specific to tendon tissue and has been identified in tendon mesenchyme (28). Tenascin C is characterized by enhanced expression levels in the tissues of the cartilage and the tendon.
Earlier reports revealed that Scx is expressed in mouse myoblast C2C12 cells (29) and normal muscles (GDS234 and GDS641), as evidenced by profiling studies in the GEO database. Therefore, these findings indicate that the expression of TENC and Scx is comparatively specific for tendon tissues.

Certain limitations existed in our investigation: i) The two-dimensional nature of the cell culture and the absence of proper matrix interactions (30), the presence of intercellular interactions (31), or the lack of mechanical stimulation (32), could lead to the loss expression of tendon-selective genes in the primary tendon cells that were formed. ii) In future experiments, substrates of collagen gel with three dimensions (30) or loads (33) are to be utilized to re-establish the mechanostat set point during the cell culture period. Mesenchymal stem cells are capable of expressing tendon markers when a cyclic load and three-dimensional culture are employed. iii) Further research is required to explore the impact of various hypoxic environments on the differentiation of MenSCs and to establish the optimal hypoxic $\mathrm{O}_{2}$ concentration stimulating the process. iv) In the future, more comprehensive in vivo investigations are necessary to determine the underlying mechanism of tenogenic differentiation.

In conclusion, in spite of the absence of a significant difference between the normoxic and hypoxic group, after 3 weeks' co-culture with ATCs, successful differentiation of MenSCs into tenogenic cells was achieved, which induced the production of the extracellular matrix that was similar to the original and led to the expression of the specific AT markers (THBS4, TENC and Scx). In future, MenSCs may be considered a potent novel source of MSCs that may contribute substantially towards the further development of therapies to treat Achilles tendinitis.

\section{Acknowledgements}

Financial support for the present study was provided by The Foundation of ZheJiang Medicine and Health Subject (grant no. 201341061) and The WenZhou Technology Bureau (grant no. Y20100357).

\section{References}

1. Carcia CR, Martin RL, Houck J and Wukich DK; Orthopaedic Section of the American Physical Therapy Association: Achilles pain, stiffness, and muscle power deficits: Achilles tendinitis. J Orthop Sports Phys Ther 40: A1-A26, 2010.

2. Khan KM, Cook JL, Bonar F, Harcourt P and Astrom M: Histopathology of common tendinopathies. Update and implications for clinical management. Sports Med 27: 393-408, 1999.

3. Knobloch K, Kraemer R, Lichtenberg A, Jagodzinski M, Gossling T, Richter M, Zeichen J, Hufner T and Krettek C: Achilles tendon and paratendon microcirculation in midportion and insertional tendinopathy in athletes. Am J Sports Med 34: 92-97, 2006.

4. Mosier SM, Pomeroy G and Manoli A II: Pathoanatomy and etiology of posterior tibial tendon dysfunction. Clin Orthop Relat Res: 12-22, 1999.

5. Chong AK, Ang AD, Goh JC, Hui JH, Lim AY, Lee EH and Lim BH: Bone marrow-derived mesenchymal stem cells influence early tendon-healing in a rabbit achilles tendon model. J Bone Joint Surg Am 89: 74-81, 2007.

6. Zantop T, Gilbert TW, Yoder MC and Badylak SF: Extracellular matrix scaffolds are repopulated by bone marrow-derived cells in a mouse model of achilles tendon reconstruction. J Orthop Res 24: 1299-1309, 2006. 
7. Wang XJ, Dong Z, Zhong XH, Shi RZ, Huang SH, Lou Y and Li QP: Transforming growth factor-beta1 enhanced vascular endothelial growth factor synthesis in mesenchymal stem cells. Biochem Biophys Res Commun 365: 548-554, 2008.

8. Luo Q, Song G, Song Y, Xu B, Qin J and Shi Y: Indirect co-culture with tenocytes promotes proliferation and mRNA expression of tendon/ligament related genes in rat bone marrow mesenchymal stem cells. Cytotechnology 61: 1-10, 2009.

9. Uysal AC and Mizuno H: Tendon regeneration and repair with adipose derived stem cells. Curr Stem Cell Res Ther 5: 161-167, 2010.

10. Gargett $\mathrm{CE}$ and Masuda H: Adult stem cells in the endometrium. Mol Hum Reprod 16: 818-834, 2010

11. Meng X, Ichim TE, Zhong J, Rogers A, Yin Z, Jackson J, Wang H, Ge W, Bogin V, Chan KW, et al: Endometrial regenerative cells: A novel stem cell population. J Transl Med 5: 57, 2007.

12. Patel AN, Park E, Kuzman M, Benetti F, Silva FJ and Allickson JG: Multipotent menstrual blood stromal stem cells: Isolation, characterization, and differentiation. Cell Transplant 17: 303-311, 2008.

13. Cui CH, Uyama T, Miyado K, Terai M, Kyo S, Kiyono T and Umezawa A: Menstrual blood-derived cells confer human dystrophin expression in the murine model of Duchenne muscular dystrophy via cell fusion and myogenic transdifferentiation. Mol Biol Cell 18: 1586-1594, 2007.

14. Jelinsky SA, Archambault J, Li L and Seeherman $\mathrm{H}$ Tendon-selective genes identified from rat and human musculoskeletal tissues. J Orthop Res 28: 289-297, 2010.

15. Livak KJ and Schmittgen TD: Analysis of relative gene expression data using real-time quantitative PCR and the 2(-Delta Delta C(T)) method. Methods 25: 402-408, 2001.

16. Cui CH, Uyama T, Miyado K, Terai M, Kyo S, Kiyono T and Umezawa A: Menstrual blood-derived cells confer human dystrophin expression in the murine model of Duchenne muscular dystrophy via cell fusion and myogenic transdifferentiation. Mol Biol Cell 18: 1586-1594, 2007

17. Cho NH, Park YK, Kim YT, Yang H and Kim SK: Lifetime expression of stem cell markers in the uterine endometrium. Fertil Steril 81: 403-407, 2004

18. Young M: Stem cell applications in tendon disorders: A clinical perspective. Stem Cells Int 2012: 637836, 2010.

19. Qiu Y, Wang X, Zhang Y, Rout R, Carr AJ, Zhu L, Xia Z and Sabokbar A: Development of a refined tenocyte differentiation culture technique for tendon tissue engineering. Cells Tissues Organs 197: 27-36, 2013.

20. Grisendi G, Annerén C, Cafarelli L, Sternieri R, Veronesi E, Cervo GL, Luminari S, Maur M, Frassoldati A, Palazzi G, et al: GMP-manufactured density gradient media for optimized mesenchymal stromal/stem cell isolation and expansion. Cytotherapy 12: 466-477, 2010.

21. Yao L, Bestwick CS, Bestwick LA, Maffulli N and Aspden RM: Phenotypic drift in human tenocyte culture. Tissue Eng 12 1843-1849, 2006.
22. Ahmed IM, Lagopoulos M, McConnell P, Soames RW and Sefton GK: Blood supply of the Achilles tendon. J Orthop Res 16: 591-596, 1998.

23. Stoyanov JV, Gantenbein-Ritter B, Bertolo A, Aebli N, Baur M, Alini $\mathrm{M}$ and Grad S: Role of hypoxia and growth and differentiation factor-5 on differentiation of human mesenchymal stem cells towards intervertebral nucleus pulposus-like cells. Eur Cell Mater 21: 533-547, 2011

24. Baumgartner L, Arnhold S, Brixius K, Addicks K and Bloch W: Human mesenchymal stem cells: Influence of oxygen pressure on proliferation and chondrogenic differentiation in fibrin glue in vitro. J Biomed Mater Res A 93: 930-940, 2010.

25. Risbud MV, Albert TJ, Guttapalli A, Vresilovic EJ, Hillibrand AS, Vaccaro AR and Shapiro IM: Differentiation of mesenchymal stem cells towards a nucleus pulposus-like phenotype in vitro: Implications for cell-based transplantation therapy. Spine (Phila Pa 1976) 29: 2627-2632, 2004.

26. Fehrer C, Brunauer R, Laschober G, Unterluggauer $H$, Reitinger S, Kloss F, Gülly C, Gassner R and Lepperdinger G: Reduced oxygen tension attenuates differentiation capacity of human mesenchymal stem cells and prolongs their lifespan. Aging Cell 6: 745-757, 2007.

27. Sodersten F, Ekman S, Schmitz M, Paulsson M and Zaucke F: Thrombospondin-4 and cartilage oligomeric matrix protein form heterooligomers in equine tendon. Connect Tissue Res 47: 85-91, 2006.

28. Baumeister A, Arber S and Caroni P: Accumulation of muscle ankyrin repeat protein transcript reveals local activation of primary myotube endcompartments during muscle morphogenesis. J Cell Biol 139: 1231-1242, 1997.

29. Liu Y, Nifuji A, Tamura M, Wozney JM, Olson EN and Noda M: Scleraxis messenger ribonucleic acid is expressed in $\mathrm{C} 2 \mathrm{C} 12$ myoblasts and its level is down-regulated by bone morphogenetic protein-2 (BMP2). J Cell Biochem 67: 66-74, 1997.

30. Lavagnino M and Arnoczky SP: In vitro alterations in cytoskeletal tensional homeostasis control gene expression in tendon cells. J Orthop Res 23: 1211-1218, 2005.

31. Banes AJ, Weinhold P, Yang X, Tsuzaki M, Bynum D, Bottlang M and Brown T: Gap junctions regulate responses of tendon cells ex vivo to mechanical loading. Clin Orthop Relat Res: S356-S370, 1999.

32. Egerbacher M, Arnoczky SP, Caballero O, Lavagnino M and Gardner KL: Loss of homeostatic tension induces apoptosis in tendon cells: An in vitro study. Clin Orthop Relat Res 466: $1562-1568,2008$

33. Tsuzaki M, Bynum D, Almekinders L, Faber J and Banes AJ: Mechanical loading stimulates ecto-ATPase activity in human tendon cells. J Cell Biochem 96: 117-125, 2005. 